\section{kompakt}

\section{Exemestan verhindert Brustkrebs}

Menopausale Frauen mit einem erhöhten Risiko für Brustkrebs profitieren von Exemestan. Der Aromatase-Inhibitor, der die Bildung von Östrogenen vermindert, senkte in einer Studie mit 4560 menopausalen Frauen die Inzidenz einer Erkrankung um 65\%. Über fünf Jahre hatten die Probandinnen täglich $25 \mathrm{mg}$ Exemestan oder ein Placebo erhalten. Allerdings traten unter Exemestan vermehrt Hitzewallungen und Arthritiden auf. Goss PE et al, N Engl J Med 2011 (online first)

\section{Herzinfarkt: Gefahr durch Mandel-Op.}

Kinder und Jugendliche, denen vor dem 20. Lebensjahr die Mandeln oder die Appendix entnommen wurden, erleiden im frühen Erwachsenenalter häufiger einen Herzinfarkt. In einer Fall-Kontroll-Studie verglichen Forscher 54.449 Blinddarm- und 27.284 Mandeloperierte mit Kontrollpersonen. Das Ergebnis: ein um 33\% erhöhtes Herzinfarktrisiko nach Appendektomie und ein um $44 \%$ erhöhtes Risiko nach Tonsillektomie.

Janszky l et al, Eur Heart J 2011 (online first)

\section{Mehr Männer durch Radioaktivität}

Radioaktivität begünstigt männliche Geburten. So konnten Forscher zeigen, dass nach dem Atomunfall in Tschernobyl 1986 in 39 europäischen Ländern relativ mehr Jungen zur Welt kamen und damit der langjährige Trend einer Abnahme des Jungenüberschusses durchbrochen wurde. Bereits 1963 hatten die Nachwirkungen zahlreicher Atomtests, die in jenem Jahr verboten wurden, zum selben Trendwechsel in den USA und Europa geführt.

Scherb H, Voigt K, Environ Sci Pollut Res 2011, 18:697

\title{
Osteoporose voraussagen?
} Mehr Falten, dünnere Knochen as

Wie hier wohl die Knochendichte aussieht?

Gesichtsfalten nach der Menopause sind nicht nur kosmetisch störend, sondern korrelieren auch mit der Dichte der Knochen. Von Bedeutung sind dabei Anzahl und Tiefe der Falten sowie die Festigkeit der Haut.

In einer Studie mit 114 Frauen im Alter von 40 bis 60 Jahren fanden Forscher heraus, dass ein inverser Zusammenhang zwischen Fältelung der Gesichtshaut und Dichte der langen Röhrenknochen besteht. Die Teilnehmerinnen hatten ihre letzte Periode innerhalb der letzten drei Jahre gehabt und erhielten keine Hormontherapie. Je tiefer und stärker die Gesichtsfalten der Probandinnen ausgeprägt waren, desto geringer fiel die Knochen- dichte aus, welche die Wissenschaftler mithilfe der Dual-Röntgen-Absorptiometrie und sonographischer Messgeräte analysierten. Faktoren wie Alter oder Körperzusammensetzung hatten keinen Einfluss auf diesen Zusammenhang.

\section{Ein Blick ins Gesicht genügt}

In Zukunft könnte eine solche Relation z.B. in puncto Osteoporose- und Frakturrisiko bedeutsam werden. Bei der Beratung postmenopausaler Frauen würde damit ein Blick ins Gesicht eine Einschätzung des Risikos ermöglichen. Allerdings spielt der Umgang der Patientin mit Kosmetika eine Rolle.

(cd) 